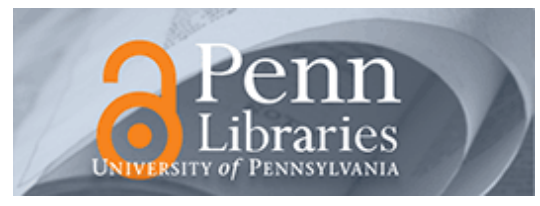

University of Pennsylvania

ScholarlyCommons

November 2007

\title{
Hydrostatic Pressure Differentially Regulates Outer and Inner Annulus Fibrosus Cell Matrix Production in 3D Scaffolds
}

Anna T. Reza

University of Pennsylvania

Steven B. Nicoll

University of Pennsylvania, nicoll@seas.upenn.edu

Follow this and additional works at: https://repository.upenn.edu/be_papers

\section{Recommended Citation}

Reza, A. T., \& Nicoll, S. B. (2007). Hydrostatic Pressure Differentially Regulates Outer and Inner Annulus Fibrosus Cell Matrix Production in 3D Scaffolds. Retrieved from https://repository.upenn.edu/be_papers/ 99

Postprint version. Published in Annals of Biomedical Engineering, online November 17, 2007.

Publisher URL: http://dx.doi.org/10.1007/s10439-007-9407-6

This paper is posted at ScholarlyCommons. https://repository.upenn.edu/be_papers/99

For more information, please contact repository@pobox.upenn.edu. 


\title{
Hydrostatic Pressure Differentially Regulates Outer and Inner Annulus Fibrosus Cell Matrix Production in 3D Scaffolds
}

\author{
Abstract \\ Mechanical stimulation may be used to enhance the development of engineered constructs for the \\ replacement of load bearing tissues, such as the intervertebral disc. This study examined the effects of \\ dynamic hydrostatic pressure (HP) on outer and inner annulus (OA, IA) fibrosus cells seeded on fibrous \\ poly(glycolic acid)-poly(L-lactic acid) scaffolds. Constructs were pressurized ( $5 \mathrm{MPa}, 0.5 \mathrm{~Hz})$ for four \\ hours/day from day 3 to day 14 of culture and analyzed using ELISAs and immunohistochemistry (IHC) to \\ assess extracellular matrix (ECM) production. Both cell types were viable, with OA cells exhibiting more \\ infiltration into the scaffold, which was enhanced by HP. ELISA analyses revealed that HP had no effect on \\ type I collagen production while a significant increase in type II collagen (COL II) was measured in \\ pressurized OA constructs compared to day 14 unloaded controls. Both OA and IA dynamically loaded \\ scaffolds exhibited more uniform COL II elaboration as shown by IHC analyses, which was most \\ pronounced in OA-seeded scaffolds. Overall, HP resulted in enhanced ECM elaboration and organization \\ by OA-seeded constructs, while IA-seeded scaffolds were less responsive. As such, hydrostatic \\ pressurization may be beneficial in annulus fibrosus tissue engineering when applied in concert with an \\ appropriate cell source and scaffold material.
}

\section{Keywords}

intervertebral disc, extracellular matrix, mechanical stimulation, collagen, tissue engineering

\section{Comments}

Postprint version. Published in Annals of Biomedical Engineering, online November 17, 2007. Publisher URL: http://dx.doi.org/10.1007/s10439-007-9407-6 


\title{
Hydrostatic Pressure Differentially Regulates Outer and Inner Annulus Fibrosus Cell Matrix Production in 3D Scaffolds
}

\author{
Anna T. Reza and Steven B. Nicoll \\ Department of Bioengineering \\ University of Pennsylvania \\ Room 240 Skirkanich Hall \\ 210 S. $33^{\text {rd }}$ Street \\ Philadelphia, PA 19104
}

Abbreviated Title: Pressure Regulates OA and IA Matrix Production in 3D

Corresponding Author:

Steven B. Nicoll, Ph.D.

Department of Bioengineering,

University of Pennsylvania

Room 240 Skirkanich Hall

210 S. $33^{\text {rd }}$ Street

Philadelphia, PA 19104

Tel: 215-573-2626

Fax: 215-573-2071

Email: nicoll@seas.upenn.edu 


\begin{abstract}
Mechanical stimulation may be used to enhance the development of engineered constructs for the replacement of load bearing tissues, such as the intervertebral disc. This study examined the effects of dynamic hydrostatic pressure (HP) on outer and inner annulus (OA, IA) fibrosus cells seeded on fibrous poly(glycolic acid)-poly(L-lactic acid) scaffolds. Constructs were pressurized $(5 \mathrm{MPa}, 0.5 \mathrm{~Hz})$ for four hours/day from day 3 to day 14 of culture and analyzed using ELISAs and immunohistochemistry (IHC) to assess extracellular matrix (ECM) production. Both cell types were viable, with OA cells exhibiting more infiltration into the scaffold, which was enhanced by HP. ELISA analyses revealed that HP had no effect on type I collagen production while a significant increase in type II collagen (COL II) was measured in pressurized OA constructs compared to day 14 unloaded controls. Both OA and IA dynamically loaded scaffolds exhibited more uniform COL II elaboration as shown by IHC analyses, which was most pronounced in OA-seeded scaffolds. Overall, HP resulted in enhanced ECM elaboration and organization by OA-seeded constructs, while IA-seeded scaffolds were less responsive. As such, hydrostatic pressurization may be beneficial in annulus fibrosus tissue engineering when applied in concert with an appropriate cell source and scaffold material.
\end{abstract}

\title{
KEY TERMS:
}

Intervertebral disc, extracellular matrix, mechanical stimulation, collagen, tissue engineering 


\section{INTRODUCTION}

The intervertebral disc (IVD) is a heterogeneous structure comprised of the outer annulus fibrosus (OA), inner annulus fibrosus (IA), and the nucleus pulposus (NP). These regions vary in both gross anatomy and function. The OA is organized into concentric lamellae, rich in type I collagen (COL I), that maintain disc shape and allow the spine to resist tensile loads ${ }^{9}$. The NP is a hydrated tissue, characterized by high proteoglycan content (i.e., aggrecan) and type II collagen (COL II) ${ }^{2}$. This gelatinous region functions to resist compressive loads through the generation of a hydrostatic swelling pressure. The IA serves as a transition zone between the lamellar structure of the OA and the less organized NP. Progressing radially from the OA to the NP, the water and proteoglycan content of the disc increase while collagen content decreases ${ }^{27}$. Together, the OA, IA, and NP permit motion and flexibility, support and distribute loads, and dissipate energy in the spine ${ }^{2}$.

IVD degeneration occurs due to the dehydration of the NP, largely from proteoglycan loss, and gives rise to increased disc stiffness and subsequent low back pain from the altered distribution of loads ${ }^{2}$. Disc degeneration is accompanied by an increase in matrix degrading enzymes such as matrix metalloprotease-3 (MMP-3), an aggrecandegrading enzyme, and MMP-13, a collagenase particularly effective at cleaving the triple helices of COL II ${ }^{7}$.

Chronic low back pain and disc degeneration are seen more frequently among those that engage in recurrent heavy lifting or experience sustained vibration in their

occupation $^{33}$. Current modes of treatment for low back pain include simple non-surgical options, such as a decrease in activity or the administration of pain relievers and anti- 
inflammatory medication ${ }^{1}$. More severe cases may require surgical intervention such as discectomy, to remove a small portion of the damaged disc in instances of disc herniation, or spinal fusion, removing an entire IVD and fusing the two adjacent vertebrae together via metal rods ${ }^{16}$. Spinal fusion results in a decreased range of motion and alters the biomechanics of the spine, possibly contributing to the subsequent degeneration of neighboring discs ${ }^{5,18,26}$. As such, tissue engineering strategies have been explored as treatment alternatives to restore both IVD structure and function.

It is well-known that the environment plays a significant role in determining cellular phenotype in the IVD ${ }^{2,8,27,29,34}$. In addition to appropriate material scaffolds, IVD tissue engineering may be enhanced through the application of mechanical loads to mimic in vivo conditions, and thereby, regulate the cellular phenotype. Deformational loading at physiologic magnitudes and frequencies has been reported to have beneficial effects, increasing production of extracellular matrix (ECM) macromolecules, including COL II and glycosaminoglycans (GAGs), and decreasing production of catabolic factors, such as MMPs ${ }^{10,13}$. Low frequency dynamic compression $(0.01 \mathrm{~Hz}, 1 \mathrm{MPa})$ in an in vivo rat tail model increased ECM gene expression in NP cells while high frequency compression $(1 \mathrm{~Hz})$ increased catabolic factor expression ${ }^{17}$. Cyclic tensile strain (1- 8\%, $1 \mathrm{~Hz}$ ) has also been shown to produce beneficial effects, increasing COL II and aggrecan gene expression while decreasing MMP-3 expression in annulus fibrosus cells encapsulated in collagen gels ${ }^{24}$.

Researchers have also investigated the effects of hydrostatic pressurization on IVD cell culture systems. Tissue-engineered constructs comprised of NP cells encapsulated in collagen or polysaccharide hydrogel scaffolds have been shown to 
respond to hydrostatic pressurization with increased production of collagen and GAGs when subjected to physiologic ranges of mechanical stimulation $(0.1-3.0 \mathrm{MPa})^{11,12,24,25}$. For example, a study by Neidlinger-Wilke et al. found that NP cells encapsulated in collagen gels increased aggrecan gene expression and decreased expression of MMP-2 and -3 in response to $0.25 \mathrm{MPa}$ hydrostatic pressure applied at a frequency of $0.1 \mathrm{~Hz}^{24}$. Although tensile strain produced through flexion, extension, and torsion of the disc is the dominant form of mechanical loading in the annulus ${ }^{2}$, this region also experiences hydrostatic pressure, in particular, in the inner region of the tissue. The few studies that have investigated the effects of hydrostatic pressure on annulus fibrosus cells encapsulated the cells in hydrogels, which may not be the most appropriate scaffold given that they normally reside in the fibrous, lamellar structure of the annulus, rather than the hydrated gel-like NP. As shown by Neidlinger-Wilke et al., annulus fibrosus cells encapsulated in collagen gels were less responsive to pressures applied in the lower physiologic range (0.25 MPa), with cells decreasing aggrecan gene expression ${ }^{24}$. Additionally, Hutton et al. noted a reduction in collagen synthesis by alginate encapsulated annulus fibrosus cells exposed to hydrostatic pressure at 0.35 and $1 \mathrm{MPa}^{11 \text {, }}$ 12. Although these results seem to imply that annulus fibrosus cells respond negatively to hydrostatic pressure, the format of the three-dimensional (3D) scaffold may play a large role in determining the cellular response to applied pressures. In particular, a polymer fiber mesh may better represent the native environment of the annulus in comparison to a hydrogel, and thus, may be more suitable for culturing annulus fibrosus cells.

Therefore, the goal of this study was to investigate the effect of hydrostatic pressurization on OA and IA cells seeded on fibrous (poly)glycolic acid/(poly)L-lactic 
acid (PGA-PLLA) scaffolds. We hypothesized that the application of hydrostatic pressure would promote production of COL II and chondroitin sulfate proteoglycan (CSPG) in IA cell-seeded constructs and would modify the phenotype of OA constructs to similarly promote COL II and CSPG production, although to a lesser degree than in IA samples.

\section{MATERIALS AND METHODS}

\section{Primary Cell Isolation and Culture}

All cell culture supplies, including media, antibiotics, and buffering agents, were purchased from Invitrogen (Carlsbad, CA) unless otherwise noted. Discs C2-C4 were isolated from bovine caudal IVDs (Moyer Packing, Souderton, PA) via sterile methods and separated into OA, IA, and NP regions through gross visual inspection based on previous studies ${ }^{3}$. Tissue was maintained in Dulbecco's Modified Eagle Medium (DMEM) supplemented with 20\% fetal bovine serum (FBS) (Hyclone, Logan, UT), $0.075 \%$ sodium bicarbonate, $100 \mathrm{U} / \mathrm{mL}$ penicillin, $100 \mu \mathrm{g} / \mathrm{mL}$ streptomycin, and 0.25 $\mu \mathrm{g} / \mathrm{mL}$ Fungizone reagent at $37^{\circ} \mathrm{C}, 5 \% \mathrm{CO}_{2}$ for two days prior to digestion to ensure no contamination occurred during harvesting. A single serum lot was used for all experiments to reduce potential variability in the cellular response.

Tissue was diced and OA and IA cells were released by collagenase (Type IV, Sigma, St. Louis, MO) digestion at an activity of $7000 \mathrm{U}$ collagenase per gram of tissue. Following incubation in collagenase, undigested tissue was removed using a $40 \mu \mathrm{m}$ mesh filter. Cells from multiple levels (C2-C4) were pooled and rinsed in PBS while maintaining separation between OA and IA cells. These primary cells were plated onto 
tissue culture flasks and designated as passage 0 . Cells were expanded twice in monolayer subculture to obtain the necessary number of cells and passage 2 cells were used in all experiments ${ }^{3}$.

\section{Scaffold Preparation and Cell Culture}

A $1.1 \mathrm{~mm}$ thick non-woven PGA fiber mesh (Biomedical Structures, Warwick, RI) reinforced with a 3\% $50 \mathrm{kDa}$ PLLA (Polysciences, Warrington, PA) solution in chloroform was fashioned into $0.833 \mathrm{~cm} \times 0.5 \mathrm{~cm}$ strips and pretreated with $1 \mathrm{~N} \mathrm{NaOH}$ and ethanol to decrease polymer hydrophobicity. Scaffolds were soaked in $70 \%$ ethanol overnight prior to cell seeding to further increase wettability and were UV sterilized. The scaffolds were then seeded with $2 \times 10^{6}$ cells in a $40 \mu \mathrm{L}$ volume of media applied directly to the polymer. $20 \mu \mathrm{L}$ of the cell suspension was applied to one face of the polymer, which was then incubated for 15 minutes at $37^{\circ} \mathrm{C}$ and $5 \% \mathrm{CO}_{2}$ to allow cells to adhere to the substrate. Scaffolds were then inverted and seeded with the remaining $20 \mu \mathrm{L}$ of cell suspension on the opposite face, similarly incubated for 15 minutes, and then flooded with media. All cultures were maintained at $37^{\circ} \mathrm{C}, 5 \% \mathrm{CO}_{2}$ in DMEM supplemented by $10 \% \mathrm{FBS}, 0.075 \%$ sodium bicarbonate, $100 \mathrm{U} / \mathrm{mL}$ penicillin, and $100 \mu \mathrm{g} / \mathrm{mL}$ streptomycin with the day of scaffold seeding designated as day 0 . At day 1 (D1), the medium was fully exchanged with vitamin C supplemented medium (DMEM with $10 \%$ FBS, $50 \mu \mathrm{g} / \mathrm{mL}$ L-ascorbic acid, $0.075 \%$ sodium bicarbonate, $100 \mathrm{U} / \mathrm{mL}$ penicillin, and $100 \mu \mathrm{g} / \mathrm{mL}$ streptomycin), which was used throughout the remainder of the study. The medium was fully exchanged daily for all cultures following mechanical loading (D3 to D14). At D7 and D14, cultures were analyzed for DNA content, COL I and COL II 
protein production, sulfated GAG content, and ECM localization. Constructs were isolated for biochemical and histological analyses four hours after mechanical loading.

\section{Mechanical Loading}

Samples were loaded at a magnitude of $5 \mathrm{MPa}$ and a frequency of $0.5 \mathrm{~Hz}$ for four hours daily based on prior studies ${ }^{20,31,36}$. Loading began at D3 and continued through D14. Nine (until day 7) or six (after day 7) cell-seeded scaffolds were transferred to UVsterilized, heat-sealed bags (Daigger, Vernon Hills, IL) filled with $10 \mathrm{~mL}$ of media during the four hour loading period, and were placed in a water-filled pressure chamber housed at $37^{\circ} \mathrm{C}$ (Figure $\left.1 \mathrm{~A}, \mathrm{~B}\right)$. Bagged control specimens were similarly placed in UVsterilized, heat-sealed bags and maintained in a vessel filled with warmed distilled water for four hours/day in the incubator that contained the pressure device, but were not subjected to mechanical stimulation. After 4 hours, all samples were removed from the heat-sealed bags and cultured in tissue culture polystyrene dishes under standard culture conditions $\left(37^{\circ} \mathrm{C}, 5 \% \mathrm{CO}_{2}\right)$ identical to those for free-swelling controls.

A custom-designed, stainless steel hydrostatic pressure device based on a prior

design was used to apply the specified dynamic loading conditions ${ }^{32}$. The device consists of a stainless steel pressure chamber filled with distilled water, connected to a stainless steel piston. The piston rod is driven via an air cylinder controlled by double acting solenoid valves in line with a compressed air source (SilentAire Technology, Houston, TX). The device was purged of air bubbles through the repeated advancement of the piston against the chamber medium. Experimental samples were placed in the chamber medium; the chamber was then filled completely and sealed. Pressure magnitude was 
specified by the user and feedback-controlled by a LabVIEW program (National Instruments, Austin, TX) custom-written for this application. Frequency was controlled by varying the inlet pressure of air to the device. Magnitude and frequency were verified using a custom-written MATLAB program. Average maximum and minimum pressures were $5.04 \pm 0.05 \mathrm{MPa}$ and $0.26 \pm 0.11 \mathrm{MPa}$, respectively, while average frequency was $0.50 \pm 0.02 \mathrm{~Hz}$. The hydrostatic pressure chamber and bagged control samples were housed in an incubator at $37^{\circ} \mathrm{C}$. The hydrostatic pressure device and a representative dynamic loading cycle are shown in Figure 1 (B and C).

\section{Biochemistry}

At D7 and D14, total protein and DNA were extracted with 3M guanidine hydrochloride/0.05M Tris- $\mathrm{HCl}$ (Invitrogen) followed by $10 \mathrm{mg} / \mathrm{mL}$ pepsin digestion. Collagen production was quantified via indirect ELISAs using monoclonal antibodies to COL I (Sigma) and COL II (II-II6B3, Developmental Studies Hybridoma Bank, University of Iowa, Iowa City, IA). Protein values for each sample were determined using a standard curve generated from bovine COL I and COL II (Rockland Immunochemicals, Gilbertsville, PA). Briefly, following digestion, samples and standards were diluted in coating buffer and plated onto 96-well plates (Nunc Maxisorp, Nalge Nunc International, Rochester, NY) overnight for sample adsorption. Wells were rinsed and non-specific binding was blocked using bovine serum albumin fraction $\mathrm{V}$ (Sigma). Primary antibody was added and allowed to adsorb overnight. The next day, secondary biotinylated antibody (Vector Labs, Burlingame CA) and a streptavidinconjugated horseradish peroxidase (R\&D Systems, Minneapolis, MN) were reacted 
followed by the addition of tetramethylbenzidine (Vector Labs) as the substrate chromagen. The reaction was stopped by the addition of $1 \mathrm{~N}$ sulfuric acid and plates were read at an absorbance of $450 \mathrm{~nm}$ (Synergy $\mathrm{HT}^{\mathrm{TM}}$, Bio-Tek Instruments, Winooski, VT).

Total sulfated GAG content was measured using the 1,9 dimethylmethylene blue (DMMB) assay ${ }^{6}$. GAG values were determined using a chondroitin-6 sulfate standard curve (Sigma). Briefly, $5 \mu \mathrm{L}$ of sample or standard were added to a 96-well plate. 200 $\mu \mathrm{L}$ of DMMB dye was added and absorbance was determined at $525 \mathrm{~nm}$.

Total DNA content was measured using the PicoGreen DNA assay ${ }^{30}$ (Molecular Probes, Eugene, OR) with calf thymus DNA as the standard. Briefly, $100 \mu \mathrm{L}$ of PicoGreen dye was mixed with $100 \mu \mathrm{L}$ of diluted sample or standard in a microplate which was then read at $480 \mathrm{~nm}$ excitation and $520 \mathrm{~nm}$ emission. Collagen and GAG data are presented normalized to DNA.

\section{Histology and Immunohistochemistry}

Samples were fixed in $4 \%$ paraformaldehyde and processed for paraffin embedding after graded serial ethanol dehydration. Samples were sectioned at a thickness of $9 \mu \mathrm{m}$, and hematoxylin and eosin staining was conducted to visualize cellular distribution in the polymer scaffolds. Immunohistochemical analysis was performed to assess ECM accumulation. Monoclonal antibodies to COL I, COL II, and CSPG (Sigma) were used. A peroxidase-based system (Vectastain Elite ABC, Vector Labs) and 3,3' diaminobenzidine as the chromagen were used to detect ECM localization. 


\section{Statistical Analysis}

A three-way ANOVA with Tukey's post-hoc test was performed to determine the effect of cell type, loading condition, and time. All statistical analyses were conducted using JMP software (Cary, NC). Significance was set at $p<0.05$. Data represent the mean \pm standard deviation $(n=4)$.

\section{RESULTS}

As shown in Figure 2, both OA and IA cells proliferated over time, indicating robust viability when seeded on the PGA-PLLA scaffold. Total DNA per scaffold increased significantly, with marked increases for all loading conditions from D7 to D14, excluding OA free-swelling controls (Figure 2). Additionally, there was no significant effect of mechanical stimulation on DNA content. Although DNA measurements indicated no overall effect of cell type or culture condition on DNA content, OA-seeded scaffolds exhibited more extensive cellular infiltration into the interior of the constructs, with the best infiltration achieved by samples subjected to $5 \mathrm{MPa}$ pressurization (Figure 3C). Free-swelling and bagged controls displayed a higher cellular concentration localized to the periphery of scaffolds with fewer cells present in the interior of the scaffold (Figure 3 A, B, D, E).

Normalized COL I production indicated no effect of loading at both early and later time points, regardless of cell type (Figure 4). Bagged control and dynamically loaded IA constructs exhibited a significant increase in COL I production from D7 to D14; however, these samples were not significantly different from each other or their corresponding free-swelling controls (Figure 4B). Normalized COL II data revealed no 
early effect of loading on OA samples at D7 (Figure 5A). However, by D14, there was a substantial increase in dynamically loaded constructs, which was significant compared to D14 bagged controls. Bagged control and free-swelling samples were not statistically different from each other at the D14 time point. Normalized COL II data for IA samples indicated no significant difference between dynamically loaded samples and controls at D14, along with no effect of time for dynamic and free-swelling samples (Figure 5B). Nevertheless, there was a significant decrease from D7 to D14 for bagged controls, as well as a marked decrease compared to free-swelling D14 specimens.

Normalized GAG data indicated no effect of mechanical stimulation on either cell type (Figure 6). There was no significant difference between OA groups (free-swelling vs. bagged control vs. dynamic) at either time point (Fig. 6A). There was also no effect of time (D7 vs. D14) on OA free-swelling and bagged controls. There was, however, a significant decrease in GAG levels for pressurized OA samples from D7 to D14. Nonetheless, this marked temporal decrease did not result in a significant difference compared to either set of controls at either time point. There was no effect of loading or time on IA samples and the corresponding controls (Figure 6B).

Immunohistochemical analyses showed no differences between groups for COL I when comparing $5 \mathrm{MPa}$ samples to free-swelling and bagged controls (data not shown). COL II staining revealed more uniform ECM elaboration by dynamically loaded samples compared to controls (Figure $7 \mathrm{C}, \mathrm{F}$ ), which was more pronounced in OA constructs (Figure 7 A-C), as dynamically loaded OA scaffolds exhibited intense COL II staining (Figures 7C). In addition, dynamically loaded samples elaborated more dense ECM, characterized by extensive collagen deposition (Figure 7C, arrows). In contrast, free- 
swelling constructs exhibited more diffuse COL II staining throughout the polymer

(Figure 7 A). Bagged controls displayed similar staining intensity as dynamically loaded samples, exceeding that of free-swelling controls, but with less extensive ECM distribution than dynamically loaded specimens. CSPG staining also confirmed enhanced infiltration and more uniform matrix distribution by mechanically stimulated constructs over controls (Figure 8).

\section{DISCUSSION}

This investigation examined the effect of hydrostatic pressurization on OA and IA cells seeded on fibrous PGA-PLLA scaffolds. To our knowledge, this is the first study to directly compare the two cell populations of the annulus fibrosus when seeded on 3-D fibrous scaffolds subjected to mechanical stimulation. Previous research has demonstrated successful culture of IVD cells on PGA-PLLA scaffolds ${ }^{4,21,22}$. Mizuno et al. were the first to use PGA-PLLA scaffolds for IVD tissue engineering and reported increases in DNA content of annulus fibrosus cells over the course of 12 weeks, verifying the feasibility of the scaffold for use in IVD tissue engineering. In our study, increases in DNA were observed for both cell types, regardless of loading condition, indicating no cell loss due to hydrostatic pressurization (Figure 2). In addition, histological analysis showed that OA and IA cells displayed a differential response when compared to each other, with more thorough cell infiltration into the scaffold by OA cells (Figure 3).

We hypothesized that the application of hydrostatic pressure would promote production of COL II and CSPG in IA-seeded constructs and would modify the phenotype of OA-seeded constructs to similarly promote COL II and CSPG production, 
although to a lesser degree than in IA samples. Although hydrostatic pressure would be expected to be more conducive to the development of IA constructs due to their proximity to the $\mathrm{NP}^{19}$, this study demonstrated enhanced ECM production and organization in OA-seeded scaffolds over IA specimens, contrary to our hypothesis. Previous studies have examined the effects of hydrostatic pressure on cells of the annulus fibrosus in 3D hydrogels, citing decreases in anabolic gene expression and collagen production in comparison to controls ${ }^{11,12,24}$. While quantitative ELISA data (normalized to DNA) showed no overall effect of cell type on collagen production (COL I: $p=$ 0.7733, COL II: $p=0.8929)$, OA-seeded scaffolds displayed more ECM accumulation (Figure 7). This may be due to the fibrous format of the PGA-PLLA scaffold which more closely approximates the native environment of the $\mathrm{OA}$, a region rich in collagen fibrils and lacking in water-retaining proteoglycans. As a result, the polymer scaffolds used in our study may be more conducive to promoting OA cell adhesion, allowing for improved cell growth and production, assembly, and accumulation of ECM components.

In this study, hydrostatic pressurization did not translate into clear quantitative differences in collagen or GAG production by mechanically stimulated samples over controls, although there was a trend for increased COL II production by pressurized OA constructs over time (Figures 5 and 6) which was not observed in IA-seeded scaffolds. Instead, $5 \mathrm{MPa}$ of dynamic hydrostatic pressure gave rise to more extensive infiltration of the deposited ECM throughout the scaffolds and increased intensity of staining, as was observed through histological and immunohistochemical analyses (Figures 3 and 7). This enhanced infiltration and matrix organization was most evident in OA constructs and was characterized by extensive collagen deposition (Figure 7). Free-swelling and bagged 
control samples exhibited modest collagen deposition limited to regions closer to the edges of the polymer. Quantitative data for GAG content showed no effect of hydrostatic pressurization on either cell type accompanied by no effect of time (Figure 6). Although these results were not anticipated and contradict our hypothesis, they are consistent with a previous study which investigated chondrocytes seeded on PGA scaffolds, wherein GAG values decreased over time, indicating that the scaffold was unable to fully retain the elaborated $\mathrm{GAGs}^{28}$. One limitation of our study is that the culture medium was not assayed for collagen and GAG content. This would help to determine if the lack of GAG accumulation seen in all culture conditions was due to a loss of elaborated GAGs or to a decrease in production over time. Immunohistochemical analyses of CSPG revealed no differences in staining intensity between culture conditions but remained consistent with H\&E and COL II histological analyses depicting greater infiltration by pressurized samples (Figure 8). Mechanical stimulation had no effect on COL I content, as verified by ELISA and immunohistochemical analyses (Figure 4). Although hydrostatic pressure has been shown to decrease COL I expression ${ }^{35,36}$, those prior studies encapsulated cells in hydrogels, which may modulate COL I transcription in response to mechanical loading differently than the fibrous polymer meshes used in this study. Material properties of the fibrous scaffold, such as stiffness and permeability, may influence the cellular response to mechanical stimulation. In addition, the osmolarity of the culture medium was not adjusted to approximate conditions found in the disc, which may also modulate the ability of cells to respond to hydrostatic pressurization ${ }^{37}$.

Although the application of $5 \mathrm{MPa}$ of hydrostatic pressure exceeds the physiologic range of pressures observed in the disc $(0.1-3 \mathrm{MPa})^{23}$, OA- and IA-seeded 
scaffolds were not responsive in a pilot study we conducted in which scaffolds were pressurized to $2 \mathrm{MPa}$ at $0.5 \mathrm{~Hz}$. No quantitative or histological effects of loading on collagen or GAG production or cellular infiltration were observed at this magnitude (data not shown). We suspect that these results may be due to signal dampening by the heatsealed bags and/or the PGA-PLLA scaffolds, resulting in OA and IA cells not experiencing the full applied pressure. This served as the motivation to increase the applied pressure to $5 \mathrm{MPa}$, which gave rise to enhanced cellular infiltration and matrix organization. Additionally, studies conducted by Neidlinger-Wilke et al. ${ }^{24}$ and Hutton et al. ${ }^{11,12}$ which encapsulated annulus fibrosus cells in collagen and alginate hydrogels, respectively, observed decreases in aggrecan gene expression and collagen synthesis when constructs were exposed to low magnitudes of pressure. Our findings at $5 \mathrm{MPa}$ were also supported by a study by Wenger et al. who examined the effects of hydrostatic pressure on porcine annulus fibrosus cells encapsulated in alginate disks ${ }^{35}$. Disks were pressurized to 1 or $3 \mathrm{MPa}$ at a frequency of $0.5 \mathrm{~Hz}$ for a single duty cycle of 3 hours. The lower magnitude resulted in an upregulation of COL I, COL II, and aggrecan gene expression, with no effect on MMP-1 and TGF- $\beta_{1}$. Pressure applied at a magnitude of 3 MPa produced a similar increase in COL II gene expression but reduced COL I, MMP-1, and TGF- $\beta_{1}$ relative to controls, thereby modifying the phenotype of the construct to a more chondrogenic profile when subjected to higher magnitude pressurization. Although there are distinct differences in experimental design (loading parameters, duty cycle, scaffold choice, cell source, etc.) between our study and that by Wenger et al., these results independently indicate that the cells of the annulus fibrosus may respond positively to hydrostatic pressure when applied at higher magnitudes. 
While there are many studies examining the effects of hydrostatic pressure on the cells of the IVD, there is yet no consensus on the most physiologically relevant loading regimen. Pressure magnitudes in previous IVD studies ranged from 0.1 to $10 \mathrm{MPa}$, while frequencies ranged from 0 to $20 \mathrm{~Hz}^{10-14}$. Duty cycles varied from a single 20 second application of pressure ${ }^{13}$ to 30 minutes/day for 1 to 9 days ${ }^{14,15,24,25}$. In our study, the stated pressure was applied at $0.5 \mathrm{~Hz}, 4$ hours/day, from day 3 of culture to day 14 . Mechanical stimulation was not applied until day 3 of culture to allow the cells time to acclimate to their environment. A similar duty cycle (4 hrs/day for 14 days) was used successfully to promote chondrogenesis of human adult mesenchymal stem cells ${ }^{20}$, suggesting that this loading protocol may be advantageous for engineering of cartilaginous tissues.

One unique facet of the study design was to include both free-swelling and bagged control samples that were maintained alongside dynamically loaded constructs. Free-swelling specimens were grown under standard culture conditions at $37^{\circ} \mathrm{C}, 5 \% \mathrm{CO}_{2}$ in tissue culture dishes with the same volume and formulation of medium as bagged control and dynamically loaded samples. Free-swelling controls allowed us to determine if the application of dynamic hydrostatic pressure is beneficial not only when compared to bagged controls, but also when compared to standard methods of cell culture, an evaluation often neglected in hydrostatic pressure studies. A comparison of free-swelling to bagged controls provided the opportunity to assess the effects of the daily handling of the scaffolds, as required for the study (i.e., daily transfer from tissue culture dishes via forceps and subsequent placement in heat-sealed bags filled with media for four hours at atmospheric pressure). Bagged controls underwent the same necessary handling as 
dynamic samples, and thus, were deprived of nutrient and waste transport as well as gas exchange for four hours a day. Therefore, bagged controls enabled us to determine the effect of loading itself when compared to constructs that underwent the same handling and preparation methods but were not subjected to mechanical stimulation. There were no significant differences between free-swelling and bagged controls for normalized collagen and GAG production for OA seeded scaffolds. Similarly, there were no significant differences in IA COL I and GAG production. IA bagged controls produced less COL II at both time points compared to corresponding free-swelling controls. However, due to the lack of clear, consistent differences between the two types of controls, we believe that the effects of daily handling are negligible and using bagged controls alone is appropriate for such experiments.

Taken together, dynamic hydrostatic pressurization was shown to enhance and modify ECM elaboration and organization by OA-seeded constructs, while IA-seeded scaffolds were not as responsive to the mechanical stimulus. As such, hydrostatic pressure may be a beneficial method of modulating cellular phenotype for annulus fibrosus tissue engineering when used in concert with an appropriate cell source and scaffold material. Future studies will concentrate on assessing the functionality of the ECM produced in response to hydrostatic pressure via mechanical testing to determine if the increase in ECM correlates to enhanced mechanical properties. Additionally, future work will investigate the expression of catabolic factors, such as MMP-3 and MMP-13, which are known to be involved in cartilaginous tissue matrix degradation and turnover. 


\section{ACKNOWLEDGMENTS}

This work was supported by an NSF Graduate Fellowship and NIH Grants DE14228 (S.

Nicoll) and EB002425 (PI: D. Elliott). The authors would also like to thank Christopher

Hee and Jay Sy for their custom-written MATLAB and LabVIEW programs.

\section{REFERENCES}

${ }^{1}$ An, H., S. D. Boden, J. Kang, H. S. Sandhu, W. Abdu, and J. Weinstein. Summary statement: Emerging techniques for treatment of degenerative lumbar disc disease. Spine 28 (15 Suppl): S24-S5, 2003.

${ }^{2}$ Buckwalter, J. A., S. D. Boden, D. R. Eyre, V. C. Mow, and M. Weidenbaum, "Intervertebral disk aging, degeneration, and herniation." In: Orthopaedic basic science, edited by J. A. Buckwalter, T. A. Einhorn, and S. R. Simon. Rosemont, IL: American Academy of Orthopaedic Surgeons, 2000, p. 557-566.

${ }^{3}$ Chou, A. I., A. Bansal, G. J. Miller, and S. B. Nicoll. The effect of serial monolayer passaging on the collagen expression profile of outer and inner anulus fibrosus cells. Spine 31: 1875-1881, 2006.

${ }^{4}$ Chou, A. I., A. T. Reza, C. S. Choug, and S. B. Nicoll. Differential collagen expression by outer and inner annulus fibrosus and nucleus pulposus cells seeded in 3-d polymer scaffolds. Trans. Orthop. Res. Soc. 31: 1243, 2006.

${ }^{5}$ Chow, D. H., K. D. Luk, J. H. Evans, and J. C. Leong. Effects of short anterior lumbar interbody fusion on biomechanics of neighbouring unfused segments. Spine 21: 549-555, 1996.

${ }^{6}$ Farndale, R. W., C. A. Sayers, and A. J. Barrett. A direct spectrophotometric microassay for sulfated glycosaminoglycans in cartilage cultures. Conn. Tiss. Res. 9: 247-248, 1982.

${ }^{7}$ Goupille, P., M. I. Jayson, J. P. Valat, and A. J. Freemont. Matrix metalloproteinases : The clue to intervertebral disc degeneration? Spine 23: 1612-1626, 1998.

${ }^{8}$ Gruber, H. E., J. E. Carl Fisher, B. Desai, A. A. Stasky, G. Hoelscher, and J. Edward N. Hanley. Human intervertebral disc cells from the annulus: Three-dimensional culture in agarose or alginate and responsiveness to tgf-ß1. Exp. Cell Res. 235: 13-21, 1997.

${ }^{9}$ Hall, S. J., In: Basic Biomechanics, Boston: McGraw-Hill, 2003, p. 276-282.

${ }^{10}$ Handa, T., H. Ishihara, H. Ohshima, R. Osada, H. Tsuji, and K. i. Obata. Effects of hydrostatic pressure on matrix synthesis and matrix metalloproteinase production in the human lumbar intervertebral disc. Spine 22: 1085-1091, 1997.

${ }^{11}$ Hutton, W. C., W. A. Elmer, S. D. Boden, S. Hyon, Y. Toribatake, K. Tomita, and G. A. Hair. The effect of hydrostatic pressure on intervertebral disc metabolism. Spine 24: 1507-1515, 1999.

${ }^{12}$ Hutton, W. C., W. A. Elmer, L. M. Bryce, E. E. Kozlowsk, S. D. Boden, and M. Kozlowski. Do the intervertebral disc cells respond to different levels of hydrostatic pressure? Clin. Biomech. 16: 728-734, 2001. 
${ }^{13}$ Ishihara, H., D. S. McNally, J. P. G. Urban, and A. C. Hall. Effects of hydrostatic pressure on matrix synthesis in different regions of the intervertebral disk. J. Appl. Physiol. 80: 839-846, 1996.

${ }^{14}$ Kasra, M., V. K. Goel, J. D. Martin, S.-T. Wang, W. Choi, and J. A. Buckwalter. Effect of dynamic hydrostatic pressure on rabbit intervertebral disc cells. J. Orthop. Res. 21: 597-603, 2003.

${ }^{15}$ Kasra, M., W. D. Merryman, K. N. Loveless, V. K. Goel, J. D. Martin, and J. A. Buckwalter. Frequency response of pig intervertebral disc cells subjected to dynamic hydrostatic pressure. J. Orthop. Res. 24: 1967-1973, 2006.

${ }^{16}$ Lavelle, W., C. Allen, and E. D. Lavelle. Invasive and minimally invasive surgical techniques for back pain conditions. Med. Clin. of N. Am. 91: 287-298, 2007.

${ }^{17}$ MacLean, J. J., C. R. Lee, M. Alini, and J. C. Iatridis. Anabolic and catabolic mrna levels of the intervertebral disc vary with the magnitude and frequency of in vivo dynamic compression. J. Orthop. Res. 22: 1193-1200, 2004.

${ }^{18}$ Maiman, D. J., S. Kumaresan, N. Yoganandan, and F. A. Pintar. Biomechanical effects of anterior cervical spine fusion on adjacent segments. Biomed. Mater. Eng. 9: 27-38, 1999.

${ }^{19} \mathrm{McNa}$ lly, D. S., and M. A. Adams. Internal intervertebral disc mechanics as revealed by stress profilometry. Spine 17: 66-73, 1992.

${ }^{20}$ Miyanishi, K., M. C. D. Trindade, D. P. Lindsey, G. S. Beaupré, D. R. Carter, S. B. Goodman, D. J. Schurman, and R. L. Smith. Effects of hydrostatic pressure and transforming growth factor- $\$ 3$ on adult human mesenchymal stem cell chondrogenesis in vitro. Tissue Eng. 12: 1419-1428, 2006.

${ }^{21}$ Mizuno, H., A. K. Roy, C. A. Vacanti, K. Kojima, M. Ueda, and L. J. Bonassar. Tissueengineered composites of anulus fibrosus and nucleus pulposus for intervertebral disc replacement. Spine 29: 1290-1297, 2004.

${ }^{22}$ Mizuno, H., A. K. Roy, V. Zaporojan, C. A. Vacanti, M. Ueda, and L. J. Bonassar. Biomechanical and biochemical characterization of composite tissue-engineered intervertebral discs. Biomaterials 27: 362-370, 2006.

${ }^{23}$ Nachemson, A., and G. Elfstrom. Intravital dynamic pressure measurements in lumbar discs. A study of common movements, maneuvers and exercises. Scand. J. Rehabil. Med. Suppl. 1: 1-40, 1970.

${ }^{24}$ Neidlinger-Wilke, C., K. Würtz, A. Liedert, C. Schmidt, W. Börm, A. Ignatius, H.-J. Wilke, and L. Claes. A three-dimensional collagen matrix as a suitable culture system for the comparison of cyclic strain and hydrostatic pressure effects on intervertebral disc cells. J. Neurosurg.: Spine 2: 457-465, 2005.

${ }^{25}$ Neidlinger-Wilke, C., K. Würtz, J. P. G. Urban, W. Börm, M. Arand, A. Ignatius, H.-J. Wilke, and L. E. Claes. Regulation of gene expression in intervertebral disc cells by low and high hydrostatic pressure. Eur. Spine J. 15: 372-378, 2006.

${ }^{26}$ Phillips, F. M., J. Reuben, and F. T. Wetzel. Intervertebral disc degeneration adjacent to a lumbar fusion: An experimental rabbit model. J. Bone Joint Surg.: Br. 84: 289-294, 2002.

${ }^{27}$ Roughley, P. J. Biology of intervertebral disc aging and degeneration: Involvement of the extracellular matrix. Spine 29: 2691-2699, 2004. 
${ }^{28}$ Seidel, J. O., M. Pei, M. L. Gray, R. Langer, L. E. Freed, and G. Vunjak-Novakovic. Long-term culture of tissue engineered cartilage in a perfused chamber with mechanical stimulation. Biorheology 41: 445-458, 2004.

${ }^{29}$ Setton, L. A., and J. Chen. Mechanobiology of the intervertebral disc and relevance to disc degeneration. J. Bone Joint Surg. Am. 88 (Suppl 2): 52-57, 2006.

${ }^{30}$ Singer, V. L., L. J. Jones, S. T. Yue, and R. P. Haugland. Characterization of picogreen reagent and development of a fluorescence-based solution assay for double-stranded DNA quantitation. Anal. Biochem. 249: 228-238, 1997.

${ }^{31}$ Smith, R. L., S. F. Rusk, B. E. Ellison, P. Wessells, K. Tsuchiya, D. R. Carter, W. E. Caler, L. J. Sandell, and D. J. Schurman. In vitro stimulation of articular chondrocyte mrna and extracellular matrix synthesis by hydrostatic pressure. J. Orthop. Res. 14: 5360, 1996.

${ }^{32}$ Takai, E., R. L. Mauck, C. T. Hung, and X. E. Guo. Osteocyte viability and regulation of osteoblast function in a $3 \mathrm{~d}$ trabecular bone explant under dynamic hydrostatic pressure. J. Bone Miner. Res. 19: 1403-1410, 2004.

${ }^{33}$ Videman, T., M. Nurminen, and J. D. G. Tourp. Lumbar spinal pathology in cadaveric material in relation to history of back pain, occupation and physical loading. Spine 15: 728-740, 1990.

${ }^{34}$ Wang, J. Y., A. E. Baer, V. B. Kraus, and L. A. Setton. Intervertebral disc cells exhibit differences in gene expression in alginate and monolayer culture. Spine 26: 1747-1751, 2001.

${ }^{35}$ Wenger, K. H., J. A. Woods, A. Holecek, E. C. Eckstein, J. T. Robertson, and K. A. Hasty. Matrix remodeling expression in anulus cells subjected to increased compressive load. Spine 30: 1122-1126, 2005.

${ }^{36}$ Wong, M., M. Siegrist, and K. Goodwin. Cyclic tensile strain and cyclic hydrostatic pressure differentially regulate expression of hypertrophic markers in primary chondrocytes. Bone 33: 685-693, 2003.

${ }^{37}$ Wuertz, K., J. P. Urban, J. Klasen, A. Ignatius, H. J. Wilke, L. Claes, and C. NeidlingerWilke. Influence of extracellular osmolarity and mechanical stimulation on gene expression of intervertebral disc cells. J Orthop Res. 25: 1513-1522, 2007. 


\section{FIGURES AND CAPTIONS}
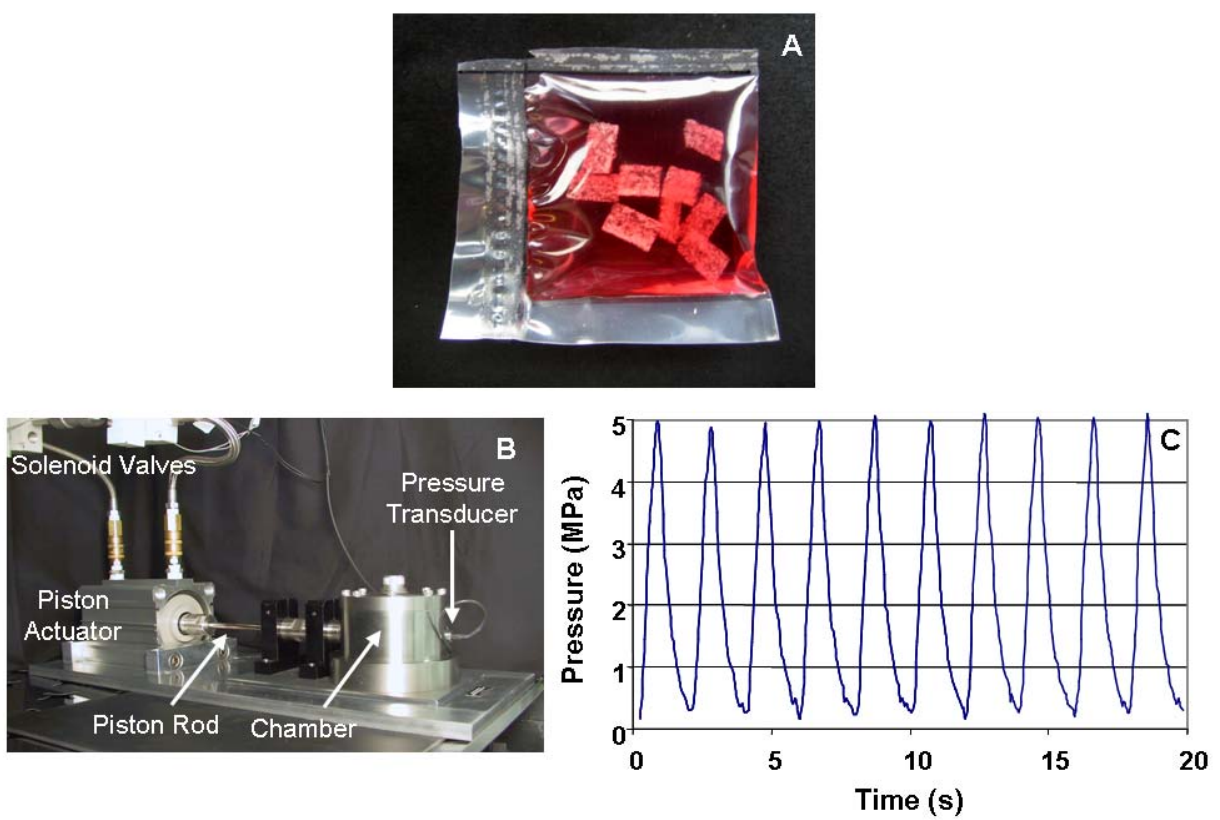

Figure 1. (A) Photomicrograph of cell-seeded scaffolds in heat-sealed bag. (B) Hydrostatic pressure device. (C) Representative 5 MPa dynamic loading cycle.

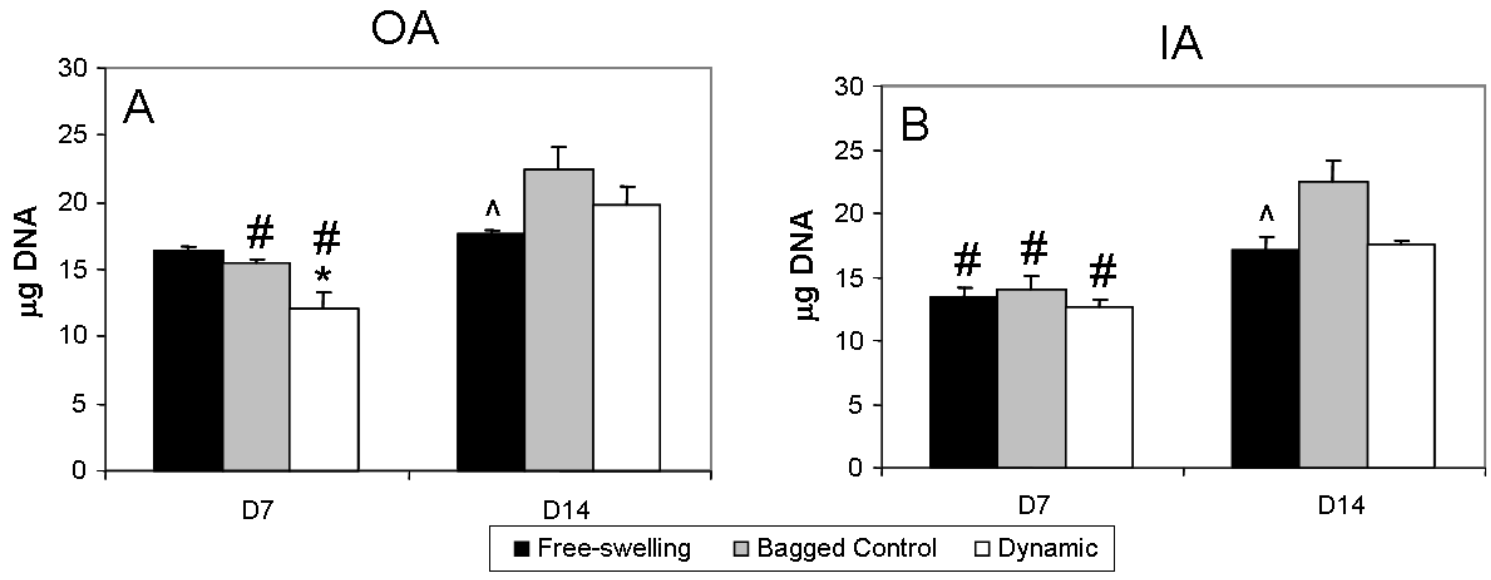

Figure 2. DNA content of outer annulus (A) and inner annulus (B) cell-seeded constructs $(n=4)$. Significance set at $\mathrm{p}<0.05$. * Significant effect of loading condition 
within time point. \# Significant effect of time within loading condition. ${ }^{\wedge}$ Significant vs. bagged control within time point.

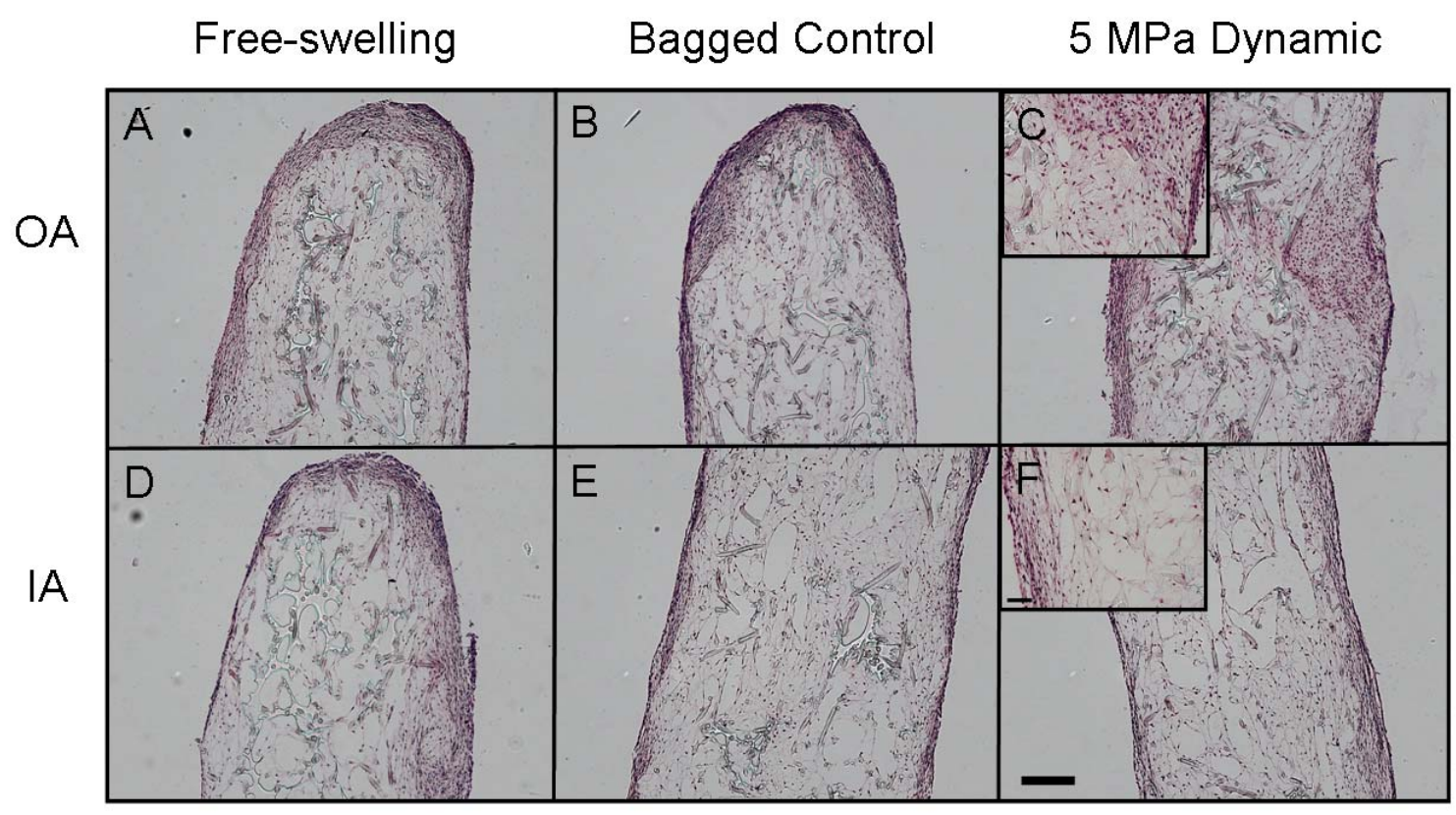

Figure 3. Hematoxylin and eosin staining of outer annulus (A-C) and inner annulus (DF) cell-seeded constructs at 14 days. Low magnification images illustrate extent of cell infiltration into the scaffold. Bar $=200 \mu \mathrm{m}$. Inlay images for $\mathrm{C}$ and $\mathrm{F}$ at higher magnification. Bar $=50 \mu \mathrm{m}$. Culture conditions indicated by column heading. 


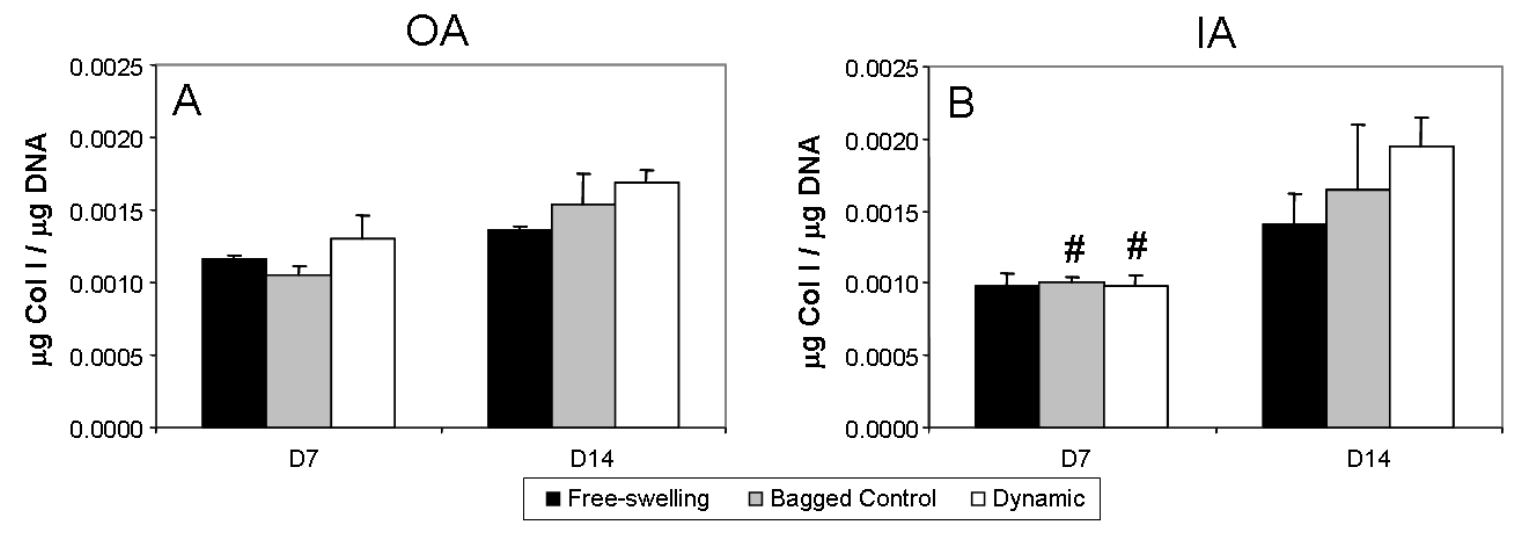

Figure 4. Normalized COL I content of outer annulus (A) and inner annulus (B) cellseeded constructs $(n=4)$. Significance set at $p<0.05$. \# Significant effect of time within loading condition.

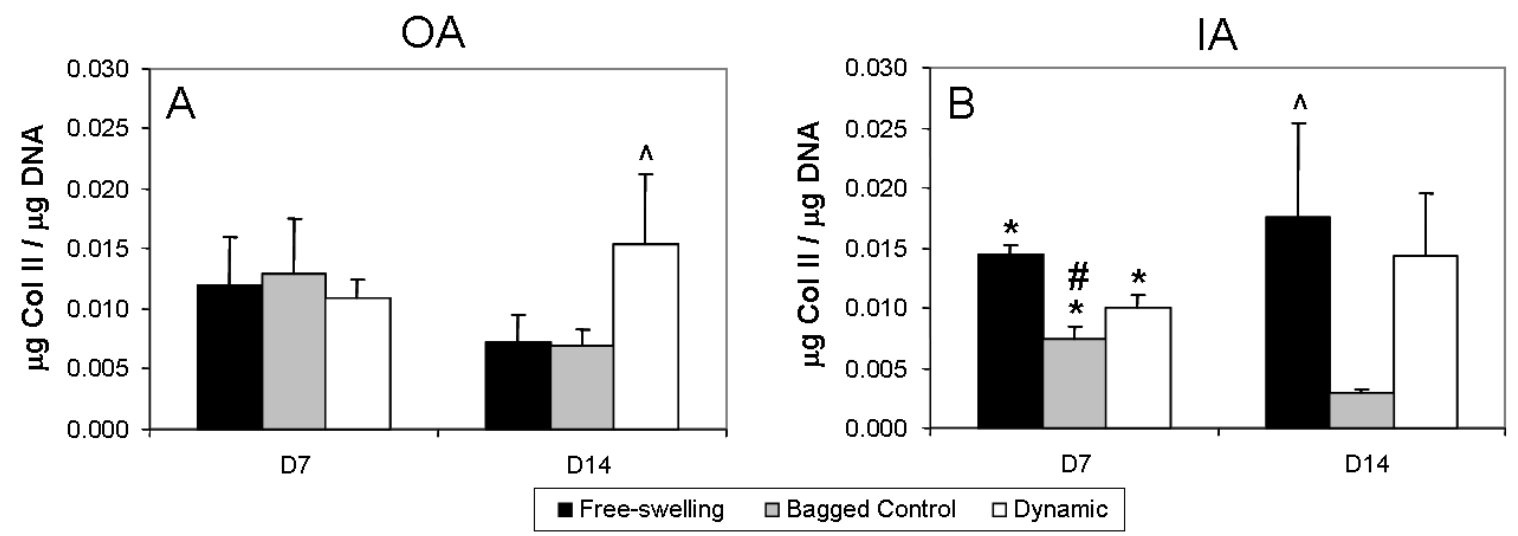

Figure 5. Normalized COL II content of outer annulus (A) and inner annulus (B) cellseeded constructs $(n=4)$. Significance set at $p<0.05$. * Significant effect of loading condition within time point. \# Significant effect of time within loading condition. ${ }^{\wedge}$ Significant vs. bagged control within time point. 


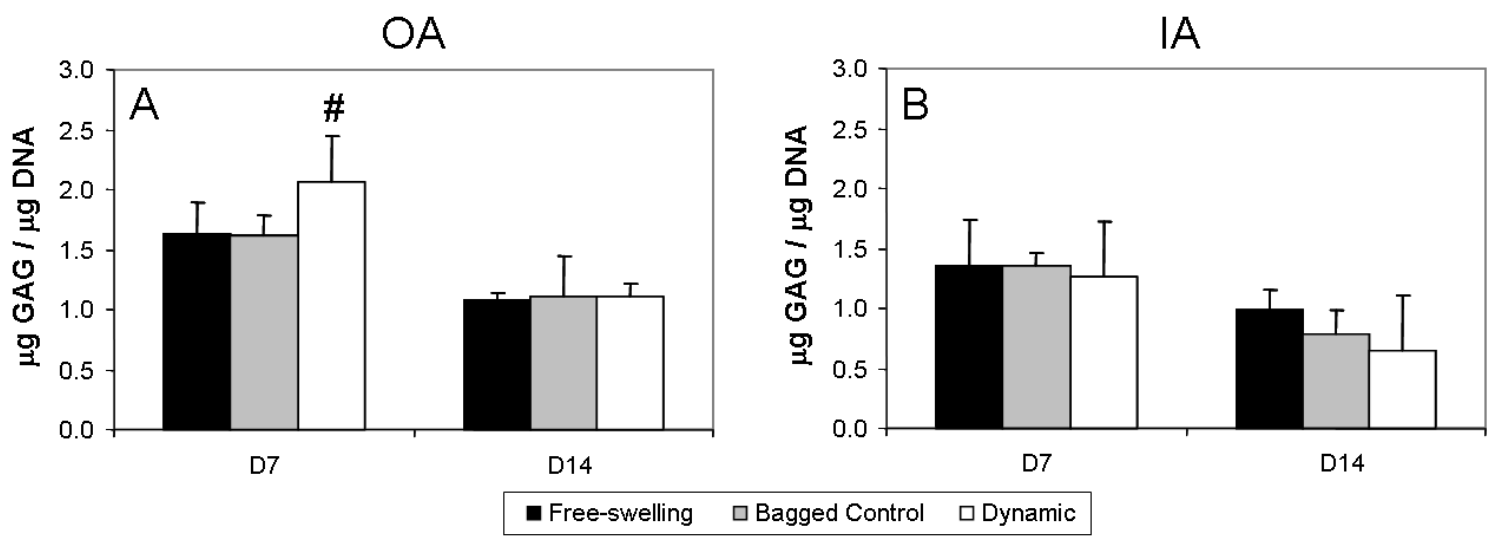

Figure 6. Normalized GAG content of outer annulus (A) and inner annulus (B) cellseeded constructs $(n=4)$. Significance set at $p<0.05$. \# Significant effect of time within loading condition.

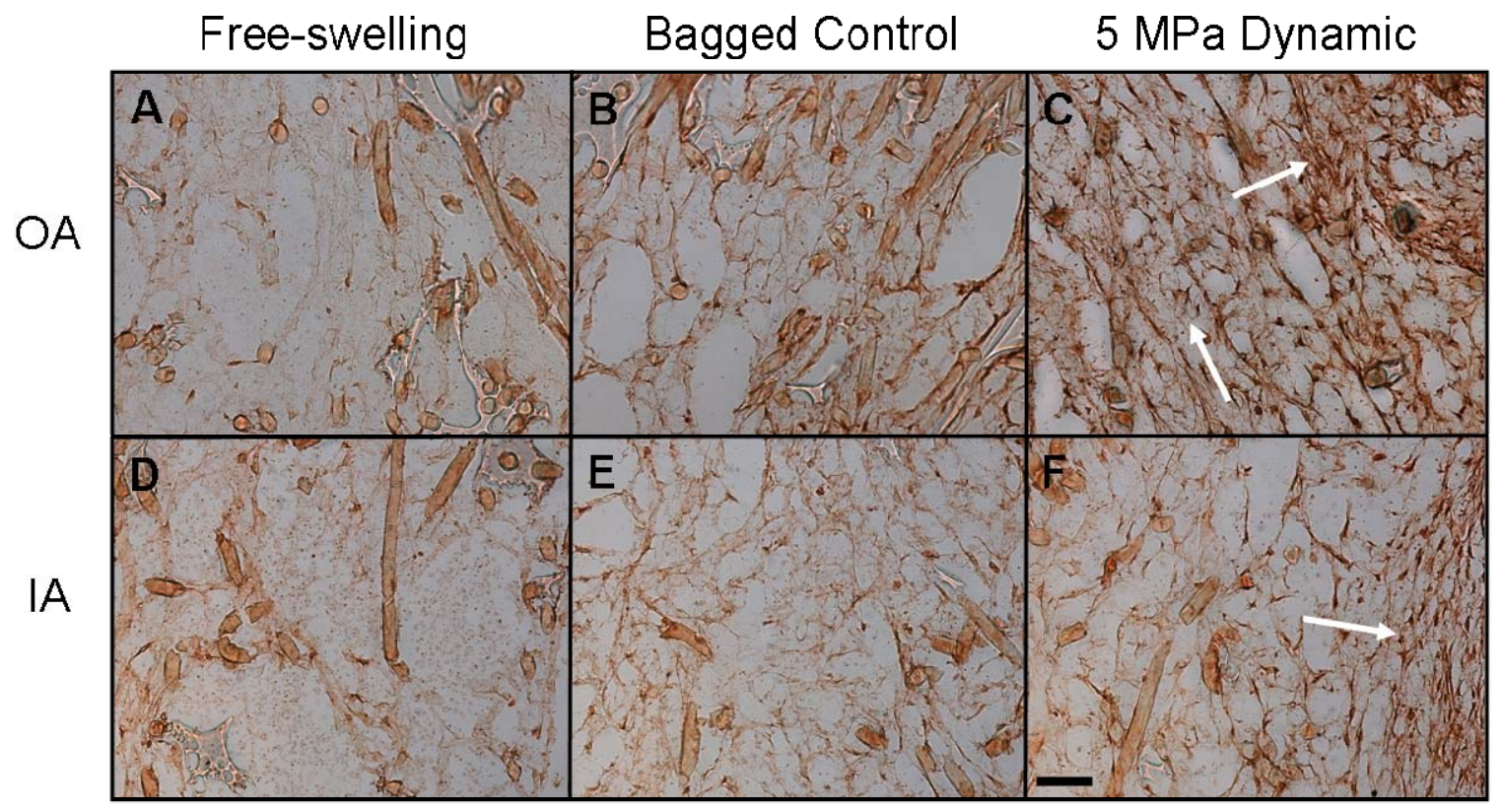

Figure 7. COL II immunohistochemical staining of outer annulus (A-C) and inner annulus (D-F) cell-seeded constructs at 14 days. Culture conditions indicated by column heading. Arrows indicate presence of collagen fibril networks, which are more pronounced in panel C. Bar $=50 \mu \mathrm{m}$. 


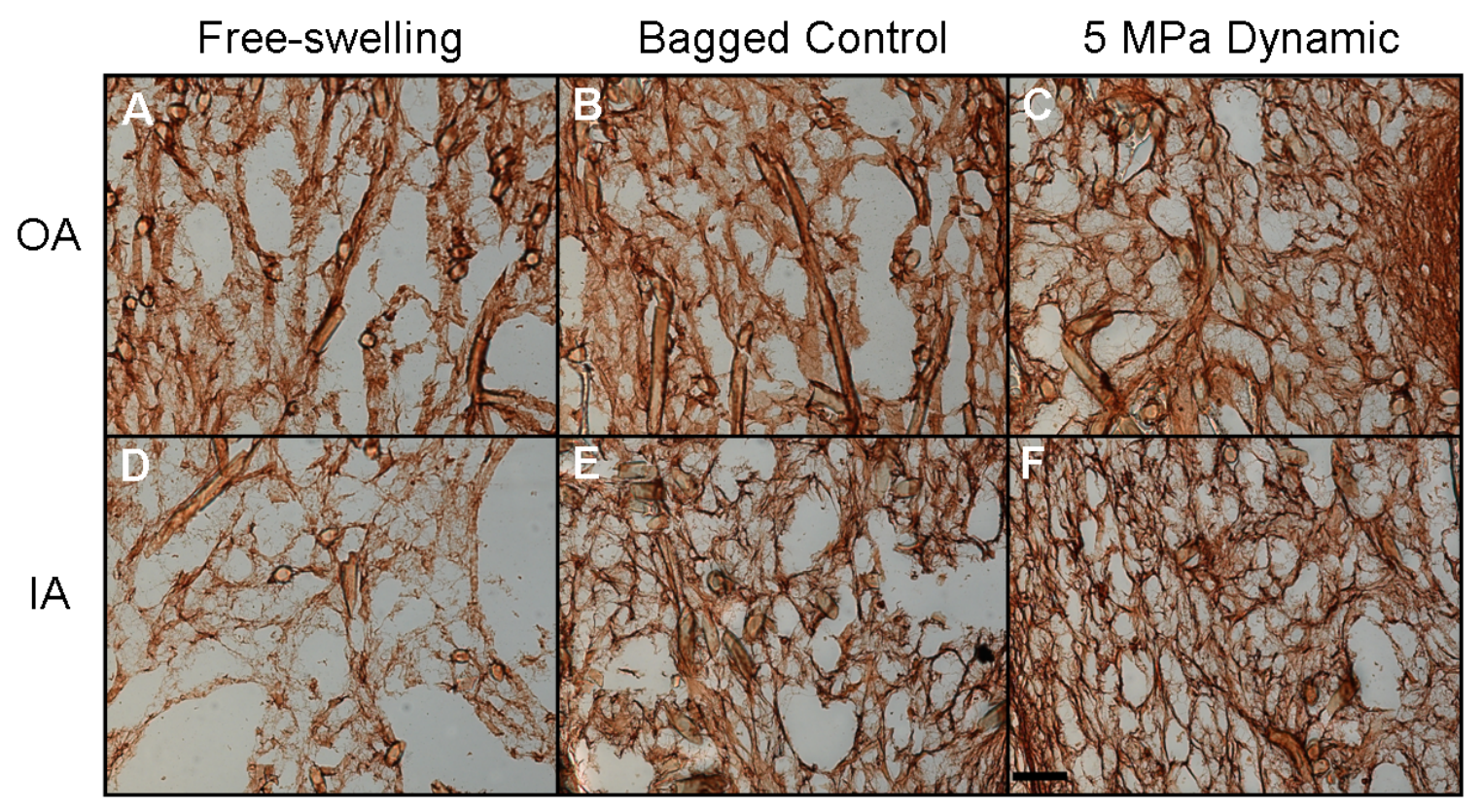

Figure 8. CSPG immunohistochemical staining of outer annulus (A-C) and inner annulus (D-F) cell-seeded constructs at 14 days. Culture conditions indicated by column heading. Bar $=50 \mu \mathrm{m}$. 